\title{
DESENVOLVIMENTO DE RECURSOS EDUCATIVOS EM MUNDOS VIRTUAIS
}

\author{
José Duarte Cardoso Gomes ${ }^{1}$, Mauro Jorge Guerreiro Figueiredo ${ }^{2}$
}

\begin{abstract}
Virtual Worlds are an important area for development and implementation of educational resources, especially by their enclosing potential in terms of interaction, collaboration, participation and immersion factors regarded as significant stimuli in the teaching-learning process. The modeling and exploration of virtual worlds can provide users innovative learning environments, as well as means to develop ideas and concepts, conveying the creation of new knowledge. The Virtual World can act as a platform for synthesis and sharing of information using various types of media. In this article we contextualize Virtual World technology applied to learning of music history, and describe the creation and implementation of a VW, developed with the program Vivaty Studio. This virtual world recreates a musical gallery, focusing on Aesthetic Periods of Music History, used in educational context, for an audience consisting of students from the $2^{\text {nd }}$ Cycle of Basic Education, in Musical Education area.
\end{abstract}

Index Terms - Virtual Worlds, VRML, X3D, Musical Education, Music History.

\section{INTRODUÇÃOO}

Atualmente, governos, companhias, instituições de educação e utilizadores são constantemente desafiados a usufruírem das vantagens e potenciais benefícios das tecnologias digitais [1].

A instrução de crianças, jovens e adultos está naturalmente associada a este fenómeno. As Tecnologias de Informação e Comunicação (TIC) permitiram o alargamento dos espaços e momentos de aprendizagem, os quais se associaram às múltiplas fontes e recursos de informação, conduzindo a uma rede alargada de aprendizagens. Segundo Costa, Peralta e Viseu [2] "o desenvolvimento da sociedade do conhecimento e a importância das TIC, não só no plano académico, mas também nos planos social, cultural, científico e económico, entre outros, fazem do conhecimento tecnológico um dos aspetos mais importantes na formação de um cidadão".

Neste contexto, as instituições de ensino viram não só uma necessidade, mas também uma oportunidade para desenvolverem novas abordagens educativas, envolvendo professores e alunos.

A utilização de Mundos Virtuais (MV) em atividades educacionais é cada vez mais frequente, em vários níveis de ensino.
Um vasto número de professores e instituições procuram e investigam ativamente formas de tornar a educação interessante e interativa, explorarando as possibilidades de aprendizagem oferecidas pelos MVs [3].

Neste artigo apresentaremos uma breve contextualização da utilização de MVs, tais como o Second Life (SL) e OpenSim (OS) no ensino.

Sintetizamos também o formato de ficheiros VRML e a norma X3D enquanto ferramentas de desenvolvimento de MVs com potencial de utilização em várias áreas, incluindo a educacional.

Descreve-se ainda a criação e implementação de um MV 3-D - Galeria Virtual -, desenvolvido com o programa Vivaty Studio, exportado no formato de ficheiros VRML, focando os Períodos Estéticos da História da Música, utilizável como recurso educativo na área do ensino e aprendizagem da Educação Musical, para um público-alvo constituído por alunos do $2^{\circ}$ Ciclo do Ensino Básico.

$\mathrm{O}$ artigo está organizado da seguinte maneira:

A seção 2 aborda a utilização de Mundos Virtuais em processos de ensino-aprendizagem, focando os Mundos Virtuais Second Life e OpenSim.

A seção 3 aborda os ficheiros VRML e a norma X3D.

Na seção 4 descrevemos o processo de desenvolvimento de um MV - Galeria Musical - e a sua aplicação em contexto educacional junto de um público-alvo constituído por alunos de Educação Musical do $2^{\circ}$ Ciclo do Ensino Básico.

Na seção 5 apresentaremos as conclusões e as principais possibilidades de trabalho futuro.

\section{MUNDOS VIRTUAIS}

Segundo Makzan [4], um Mundo Virtual é um ambiente digital semelhante ao mundo real, onde os utilizadores podem caminhar numa cidade, voar num jato de combate, conquistar um planeta ou apenas interagir entre si. Os MVs podem ser utilizados em diferentes áreas, tais como o mundo dos negócios, os jogos ou a educação. Permitem também desenvolver trabalho colaborativo com outros alunos, realizando tarefas propostas pelos professores.

As aprendizagens decorrem de fatores como imersão, navegação, exploração, interação e realização de tarefas.

Os Mundos Virtuais evoluiram desde o seu início na idade dos Multi-User Dungeons (MUDs), de um início humilde com interações baseadas em texto até aos espaços

\footnotetext{
${ }^{1}$ José Duarte Cardoso Gomes, Centro de Investigação em Artes e Comunicação, Universidade Aberta, Universidade do Algarve, jdgomes65 @ gmail.com

${ }^{2}$ Mauro Jorge Guerreiro Figueiredo, Centro de Investigação Marinha e Ambiental, Centro de Investigação em Artes e Comunicação, Instituto Superior de

Engenharia, Universidade do Algarve, mfiguei@ualg.pt
} 
digitais 3-D atuais com milhões de utilizadores e políticas e comportamentos sociais complexos.

Segundo Bell e Robbins, citados em Peachey, Gillen [5], os MVs apresentam as seguintes caraterísticas:

1. São persistentes. Eles existem independentemente de um utilizador estar ligado ou não;

2. Existem em wide area networks (WAN). Para atingir a escala de mundo, e não apenas de ambiente ou espaço, um mundo virtual deve ser acessível numa larga escala e não estar contido por uma firewall ou limitações semelhantes;

3. São massivamente multiutilizadores. Esta é uma diferença importante entre espaços virtuais construídos para alguns utilizadores e mundos que podem acomodar utilizadores a uma escala global;

4. Utilizam avatares para representar os utilizadores. Os avatares são agentes semiautónomos representados no espaço digital, capazes de realizar ações comandadas pelo utilizador. Diferenciam-se dos ícones ou perfis que representam um utilizador mas não podem realizar ações [5].

A utilização de MVs para ensinar e aprender tem atraído uma atenção crescente. Muitas universidades têm uma presença virtual em ambientes como o Second Life, mas existe pouca orientação sobre o modo como os educadores podem utilizar da maneira mais eficaz os recursos oferecidos. A natureza tridimensional (3-D) destes ambientes permite atividades e interações que não são possíveis em ambientes bidimensionais (2-D) e podem proporcionar uma plataforma para atividades que não são facilmente acomodadas num ambiente de sala de aula convencional. Contudo, apesar de serem utilizados para fins educacionais e de pesquisa em muitas instituições de ensino, é evidente que representam ainda uma minoria de educadores, turmas e projetos [6].

$\mathrm{Na}$ atualidade, não existem diretivas concretas sobre como ensinar em MVs. Estudos de caso efetuados em mundos ativos sugeriram modos de desenhar um ambiente para um mundo virtual, mas não contemplaram questões de design instrucional.

Persiste a noção que a utilização de MVs nos processos de ensino-aprendizagem é um percurso que envolve a utilização do conhecimento e experiência derivada das teorias de aprendizagem e de experiências conduzidas em ambientes educacionais, implicando um confronto com o desconhecido e o desenvolvimento de novas abordagens ligadas ou não ao conhecimento e experiência prévio.

Neste contexto, são território inexplorado tanto para professores como alunos e representam uma nova experiência de aprendizagem, não isenta de riscos [6].

\subsection{Second Life}

No Second Life, instituições de educação como universidades, escolas e associações, criaram ilhas privadas e ambientes imersivos, onde os alunos podem experimentar novos designs na construção de edifícios, familiarizarem-se com os procedimentos de uma sala de operações, conduzir experiências perigosas em segurança, aprender como planear eventos ou explorar a história em 3-D [7]. As atividades educacionais implementadas no SL podem assumir diversas formas:

- Aquisição prática de conhecimentos;

- Colaboração, trabalho de grupo e ensino à distância (elearning);

- Construção de uma comunidade;

- Descoberta, inquérito e discussão; simulação e resolução de problemas;

- Motivação;

- Criação de ambientes de aprendizagem interativos e hiperligação a ambientes de aprendizagem virtual.

Simulação, jogos e role-playing são algumas formas de explorar o potencial oferecido por este mundo virtual interativo. Os projetos educacionais associados ao SL englobam cenários de suporte ao ensino presencial tradicional, até ao ensino à distância, conduzido exclusivamente no MV [8].

Tendo em conta a grande popularidade do SL, o seu potencial, usabilidade e interface gráfica, pareceria ser a opção lógica para desenvolver e implementar um MV em contexto educativo. Contudo não é uma opção isenta de investimento. As contas livres são muito limitadas o que compromete o desenvolvimento de projetos educativos, normalmente limitados em termos de recursos económicos. Em adição, esta plataforma sendo proprietária limita a portabilidade dos mundos criados e não sabemos se continuará a existir futuramente.

\subsection{OpenSim}

Como alternativa ao SL, muitos educadores têm vindo a migrar para o OpenSimulator, ou simplesmente OpenSim. Esta plataforma teve o seu início em 2007 com o objetivo de proporcionar uma plataforma flexível e modular para a criação de ambientes virtuais personalizados. É distribuído sobre a licença BSD (Berkeley Software Distribution) sendo um programa de código aberto (opensource). Tal como o SL, o OpenSim possui um número de funcionalidades como comunicação entre residentes, modelação gráfica de objetos tridimensionais e possibilidade de interação com o ambiente virtual. Entre essas funcionalidades destacam-se as seguintes:

- Comunicação entre residentes através de chats, mensagens instantâneas ou áudio conferência;

- Modelagem gráfica de objetos 3-D, utilizada na criação de objetos tridimensionais;

- Interação com o ambiente, tornada possível pela atribuição de comportamentos aos objetos presentes no 
ambiente, ação realizada através de scripts $^{3}$, com linguagem de programação própria;

- Importação de arquivos multimédia, nomeadamente áudio, vídeo e animações;

- Captura de ecrãs, permitindo captar imagens do ambiente virtual;

- Teletransporte, através do qual os residentes se podem deslocar para diversas localidades do ambiente apenas com um clique [9].

A tabela 1 sintetiza as principais caraterísticas do SL e OpenSim:

TABELA 1

ESTUDO COMPARATIVO ENTRE SL E OPENSIM [9]

\begin{tabular}{lcc}
\hline Caraterísticas & \multicolumn{2}{c}{ Mundos Virtuais } \\
\hline Licença & Second Life & OpenSim \\
\hline $\begin{array}{l}\text { Linguagem de } \\
\text { programação do servidor }\end{array}$ & Proprietária & Código aberto \\
\hline $\begin{array}{l}\text { Linguagens de } \\
\text { programação de scripts } \\
\text { suportadas }\end{array}$ & LSL & C\# \\
\hline Criação de objetos & LSL; OSSL; C\#; \\
\hline Ocupação de terrenos & Limitado & Livire \\
\hline $\begin{array}{l}\text { Chats e mensagens } \\
\text { instantâneas }\end{array}$ & Sim & Livre \\
\hline Áudio-conferência & Sim & Sim \\
\hline
\end{tabular}

Quer o SL, quer o OpenSim revelam potencial para criação de MVs utilizáveis em processos de ensinoaprendizagem.

\section{VRML E X3D}

\subsection{VRML}

Ficheiros no formato VRML (Virtual Reality Modeling Language) revelam-se como adequados para realizar descrições estáticas ou dinâmicas de objetos 3-D, conteúdos multimédia e hiperligações. Browsers e ferramentas de autoria estão disponíveis para diversas plataformas. O VRML tem um vasto campo de utilização, incluindo aplicações de entretenimento, distribuição de visualizações, interfaces de utilizador 3-D, ambientes de trabalho colaborativo, simulações interativas em contextos educacionais, museus e lojas virtuais, entre outros [10].

$\mathrm{O}$ formato VRML possui um conjunto de recursos que o tornam particularmente atrativo para a criação de MVs. Entre estes destacam-se os seguintes: Cenário gráfico; processamento de eventos; comportamentos; encapsulamento e reutilização; conteúdo distribuído; extensibilidade; interatividade e animação.

O VRML foi concebido para ser minimalista, e no entanto completo, com complexidade suficiente para descrever

\footnotetext{
${ }^{3}$ Programa ou sequência de instruções executada por um interpretador,
}

mundos 3-D complexos. Como desvantagem, os ficheiros VRML tendem a ser bastante grandes [10].

\subsection{X3D}

O sucessor do VRML designa-se por X3D (extensible 3-D) e representa um padrão aberto para distribuir conteúdos 3-D, sendo um padrão isento de direitos de autor. Os padrões ISO (International Standards Organization) ratificados pela norma X3D proporcionam um ambiente robusto, adequado para a armazenagem, recuperação e reprodução de gráficos 3$\mathrm{D}$ em tempo real, em diversas plataformas.

O padrão evoluiu a partir do formato VRML, sendo consideravelmente mais avançado. Foi desenhado para permitir intercâmbio e interoperabilidade permitindo construir modelos 3-D simples ou sofisticados e proporciona capacidades gráficas que vão ao encontro da maioria das abordagens industriais. Com o recurso a tradutores, é possível realizar a importação e exportação de uma variedade de formatos gráficos.

Ambientes 3-D virtuais, interativos e animados podem ser construídos no X3D, reunindo os progressos mais recentes das plataformas gráficas. A possibilidade de compressão de ficheiros e a segurança de dados são outras das suas caraterísticas marcantes. A distribuição em rede é opcional, permitindo que as cenas X3D funcionem através de servidores http, em discos locais, CD's ou mesmo como anexos autónomos de email ou chat [11].

Existem grandes e pequenas diferenças entre ficheiros no formato VRML e o padrão X3D. Este tem a vantagem de utilizar o melhor do VRML, otimizando alguns dos aspetos menos positivos, nomeadamente:

- O X3D é uma versão consideravelmente mais refinada e amadurecida que o VRML;

- É compatível com o VRML. Inclui codificação XML para uma integração facilitada com outras aplicações;

- As cenas e ambientes 3-D funcionam previsivelmente em diferentes browsers e players;

- $\mathrm{O} X 3 \mathrm{D}$ é componencial, isto é, permite especificações e perfis especialmente adaptados a determinados segmentos de mercado, como aplicações CAD, médicas ou de visualização;

- A autoria de conteúdos em X3D para qualquer player é mais simples e consistente;

- Possui inúmeras caraterísticas, muitas delas herdadas do VRML plenamente integradas na estrutura da aplicação, o que torna aceitável encarar o X3D como VRML3;

- Está em constante evolução, sendo melhorado e atualizado;

- As aplicações X3D podem ser classificadas como fiáveis e previsíveis;

- Está disponível uma aplicação opensource como recurso para programadores;

\footnotetext{
${ }^{4} \mathrm{O}$ recurso de voz não está integrado no programa, portanto é necessária instalação de um módulo de voz adicional
} 
- O formato binário X3D oferece potencial de encriptação e compressão, contribuindo para maior segurança e velocidade [11].

\section{GALERIA VIRTUAL}

Neste contexto de tecnologias, foi criado um mundo virtual de ensino-aprendizagem designado Galeria Virtual, direcionado para um público-alvo de alunos do $2^{\circ}$ Ciclo do Ensino Básico, na área de Educação Musical. O MV foi desenvolvido com o programa Vivaty Studio, posteriormente exportado como ficheiro VRML e disponibilizado através da Web. Foram observadas as seguintes fases no processo de criação: Escolha da plataforma; instalação e configuração; modelagem e aplicações de ensino.

\subsection{Escolha da plataforma}

O passo inicial para a criação do ambiente de aprendizagem consistiu na escolha da plataforma. Como descrito no ponto 2, apresentavam-se como possibilidades o SecondLife e OpenSim, ambos com potencial para desenvolvimento de ambientes de aprendizagem. Tendo em conta a pequena dimensão e o número relativamente reduzido de utilizadores, optou-se pelo formato de ficheiros VRML, o qual, apesar de minimalista, é contudo um meio eficaz para descrever ambientes virtuais complexos. O programa de criação escolhido para a modelação 3-D foi o Vivaty Studio, devido à sua acessibilidade, baixo investimento e simplicidade de utilização.

\subsection{Instalação e configuração}

Tendo sido escolhido o programa, foi instalado num computador. $O$ processo de instalação é simples e não exige conhecimentos avançados em termos informáticos. Foram escolhidos os diretórios para a localização dos ficheiros multimédia fonte bem como para os ficheiros VRML. O MV foi denominado Galeria Virtual e descreve-se no ponto seguinte.

\subsection{Modelagem do MV}

A preparação dos conteúdos musicais e a modelagem 3-D consumiram grande parte do processo de criação. Na fase inicial determinou-se o tema e público-alvo, os conteúdos a apresentar e a distribuição no espaço virtual.

O tema foca os Períodos Estéticos da História da Música e destina-se a um público-alvo de alunos do $2^{\circ}$ Ciclo do Ensino Básico na área da Educação Musical. Os Períodos Estéticos da História da Música fazem parte das Metas Curriculares definidas pelo Ministério da Educação e Ciência ${ }^{5}$, no domínio da Compreensão das Artes nos Contextos e Culturas Musicais.

\footnotetext{
${ }^{5}$ http://dge.mec.pt/metascurriculares/index.php?s=directorio\&pid=2, consultado em 20-10-2013
}

A distribuição dos conteúdos no espaço tridimensional pretendia simular uma área de exposição semelhante a um museu ou a uma galeria de arte no mundo real. Assim, seria criado um conjunto de salas temáticas, cada uma albergando um dos períodos estéticos. As salas estariam ligadas por um corredor central, a partir do qual o utilizador navega, abrindo as respetivas portas. Cada sala inclui áreas definidas, entre as quais se destacam:

- Um expositor central onde são exibidos os compositores mais relevantes de cada período estético;

- Um painel onde se exibe o friso cronológico dos compositores desse período (na parede à esquerda da entrada);

- Um painel onde se exibem os instrumentos musicais mais comuns nesse período (na parede à direita da entrada);

- Um expositor para o instrumento musical mais importante do período estético, representado por um modelo 3-D, com o qual o utilizador pode interagir (rodar, ouvir, aceder a informação adicional através de hiperligações).

Estando completa a estrutura virtual da galeria, o passo seguinte consistiu na elaboração e recolha dos conteúdos multimédia a expor, bem como o tipo de interatividade. Relativamente aos conteúdos multimédia, definiu-se que o protótipo inicial deveria conter, para cada período estético:

- Compositores: Imagens, biografias, partituras, excertos de obras musicais e hiperligações;

- Instrumentos musicais: Imagens, excertos musicais, modelos 3-D, hiperligações;

- História da Arte - Pintura: Obras relevantes para expor em cada sala temática ${ }^{6}$.

A interatividade com os objetos expostos foi implementada através de botões ativados com um clique do dispositivo apontador. Está disponível, no protótipo inicial, na ativação das portas, no expositor central das salas temáticas e no instrumento 3-D mais significativo (figura 1):
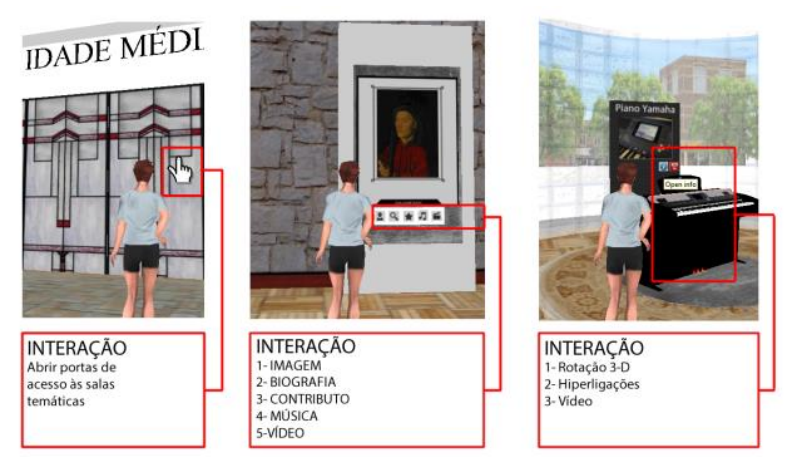

FIGURA. 1

\footnotetext{
${ }^{6}$ A presença de outras formas de arte contemporâneas visa uma melhor contextualização cultural pelos alunos do período estético.
} 
INTERAÇÕES NO MV

\subsection{Aplicações de Ensino para o MV}

A Galeria Virtual foi utilizada em contexto educativo, no ano letivo de 2012-2013, no Agrupamento de Escolas de Padrão da Légua, na EB de Leça do Balio com alunos do $2^{\circ}$ Ciclo do Ensino Básico. No dia 24 de abril, realizou-se um Peddypaper a nível de estabelecimento de ensino, e a Galeria Virtual era uma das estações do percurso. Na estação foram disponibilizados um computador multimédia com acesso à internet, um projetor e um espaço com mesas e cadeiras. $\mathrm{Na}$ estação, o professor responsável deu a escolher aos alunos um conjunto de envelopes, cada um contendo uma diferente tarefa $^{7}$ a realizar. Recebida e percebida a tarefa, o chefe do grupo, com as orientações dos colegas, navegou com o avatar no espaço virtual até à concretização do objetivo.

Todos os grupos participantes superaram as tarefas. $\mathrm{O}$ entusiasmo e motivação foi evidente.

\section{Conclusões e Trabalho Futuro}

No campo educacional, a utilização de MVs para ensinar e aprender tem despertado uma atenção crescente, embora não existam orientações precisas sobre o modo mais eficaz de explorar o potencial destes ambientes. Contudo, parece ser consensual o seu potencial no que concerne à interatividade comparativamente com ambientes virtuais 2-D, ou até como suporte a atividades difíceis de acomodar numa sala de aula convencional.

Os Mundos Virtuais Second Life, proprietário, e o OpenSim, de código-aberto, apresentam-se como plataformas com potencial para o desenvolvimento de atividades educacionais e partilham um conjunto de caraterísticas semelhantes. Muitos educadores têm migrado para o OpenSim devido ao facto de ser código-aberto, de utilizar um maior número de linguagens de programação e não ter as mesmas limitações que o SL em termos de criação de objetos e ocupação de terrenos. Como alternativa ao Second Life e OpenSim, é possível criar ambientes complexos em formato de ficheiros VRML, ou segundo as normas X3D, recorrendo a programas e browsers de internet.

Neste contexto, optou-se pelo formato de ficheiros VRML recorrendo ao programa Vivaty Studio para o desenvolvimento da Galeria Virtual.

A galeria foi utilizada em contexto educativo, no Agrupamento de Escolas de Padrão da Légua, na EB de Leça do Balio no ano letivo 2012-2013, tendo sido observados significativos níveis de participação e motivação pelos alunos envolvidos na atividade.

Estudos recentes revelam que, embora não esteja claramente provado que a utilização das TIC nos processos de ensino-aprendizagem contribua para um ensino e uma

\footnotetext{
${ }^{7}$ Por exemplo: Localizar no mundo virtual um instrumento de sopro caraterístico da Idade Média.
}

aprendizagem mais eficaz, verifica-se que conseguiram melhorar os níveis de participação e colaboração dos alunos nas aprendizagens, através de, entre outras, jogos e atividades imersivas como mundos virtuais experienciados através de avatares [12].

$\mathrm{Na}$ atualidade não existem diretivas sobre como ensinar e aprender em mundos virtuais, sendo um território inexplorado tanto para professores como para alunos e um campo propício para investigação.

Nesse sentido, julga-se pertinente aprofundar o estudo da aplicação deste tipo de recursos explorando o seu potencial didático e pedagógico como suporte ao ensino da história da música. $\mathrm{O}$ estudo das contribuições para o processo de ensinoaprendizagem decorrentes da utilização deste recurso em contexto educativo poderá constituir uma proposta de trabalho futuro.

\section{REFERÊNCIAS}

Babin, G., K. Stanoevska-Slabeva, and P. Kropf, eds. ETecnologies: Transformation in a Connected World. 2011, Springer-Verlag: Heidelberg.

Costa, F.A., H. Peralta, and S. Viseu, eds. As TIC na Educação em Portugal. Mundo de Saberes. 2007, Porto Editora, Lda.: Porto. Robbins, S. and M. Bell, Second Life for Dummies. 2007, Hoboken, NJ: Wiley Publishing, Inc.

Makzan, Flash Multiplayer Virtual Worlds. 2010, Olton, Birmingham: Packt Publishing, Ltd.

Peachey, A., et al., eds. Researching Learning in Virtual Worlds. 2010, Springer: London.

Thackray, L., J. Good, and K. Howland, Learning and Teaching in Virtual Worlds: Boundaries, Challenges and Opportunities, in Researching Learning in Virtual Worlds, A. Peachey, et al., Editors. 2010, Springer: London.

Talab, R.S. and H.R. Botterbusch, Ethical and Legal Issues in Teaching and Learning in Second Life in a Graduate Online Course, in Cases on Collaboration in Virtual Learning Environments: Processes and Interactions D. Russel, Editor. 2010, IGI Global: United States of America.

Moschini, E., The Second Life Researcher Toolkit - An Exploration of Inworld Tools, Methods and Approaches for Researching Educational Projects in Second Life, in Researching Learning in Virtual Worlds, A. Peachey, et al., Editors. 2010, Springer: London.

Freire, A., C. Rolim, and W. Bessa, Criação de um Ambiente Virtual de Ensino-Aprendizagem usando a Plataforma OpenSimulator, 2010: V CONNEPI - 2010.

Taubin, G., et al., Geometry Coding and VRML. Proceedings of the IEEE, 1998. 86(6): p. 1228-1243. Authors. 2007, San Francisco, CA: Elsevier Inc.

Aukrust, V.G., ed. Learning and Cognition in Education. 2011, Elsevier, Ltd.: Oxford. 